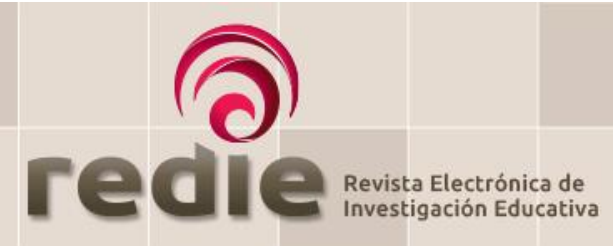

ISSN: $1607-4041$

http://redie.uabc.mx

Vol. 19, Núm. 3, 2017

\title{
Agenda actual en investigación en Didáctica de las Ciencias Naturales en América Latina y el Caribe
}

\author{
Current Agenda in Teaching Research in the Natural Sciences \\ in Latin America and the Caribbean
}

María Cristina Iturralde $\left(^{*}\right)$ citurral@fio.unicen.edu.ar
Bettina Mariel Bravo $\left(^{*}\right)$ bbravo@fio.unicen.edu.ar
Ariadna Flores (*) floresariadna2014@gmail.com

(*) Universidad Nacional del Centro de la Provincia de Buenos Aires

(Recibido: 20 de enero de 2015; Aceptado para su publicación: 7 de diciembre de 2015)

Cómo citar: Iturralde, M. C., Bravo, B. M. y Flores, A. (2017). Agenda actual en investigación en didáctica de las Ciencias Naturales en América Latina y el Caribe. Revista Electrónica de Investigación Educativa, 19(3), 49-59. https://doi.org/10.24320/redie.2017.19.3.905

\section{Resumen}

En este trabajo se presenta una revisión bibliográfica de revistas de investigación en Didáctica de las Ciencias en América Latina y el Caribe con el objetivo de identificar, describir y caracterizar las principales líneas de investigación. La base documental está conformada por 15 revistas científicas y 490 artículos que reportan trabajos de investigación educativa. De los resúmenes de los artículos se identifican las problemáticas abordadas que se agruparon en cinco dimensiones: aprendizaje de las Ciencias, enseñanza de las Ciencias, currículum de Ciencias, profesorado de Ciencias y estudiantes de profesorado de Ciencias. Los resultados revelan que la mayoría de las investigaciones que se están realizando en Latinoamérica y el Caribe tienen como problemática central la enseñanza y el aprendizaje de las ciencias.

Palabras clave: Investigación educativa, enseñanza de las ciencias, Ciencias Naturales.

\section{Abstract}

This work presents a literature review of research journals in science teaching in Latin America and the Caribbean with the aim of identifying, describing and characterizing the main lines of research. The documentary basis is made up of 15 scientific journals and 490 papers that report work in educational research. From the abstracts of the papers it was possible to identify the problems addressed, which were grouped into five dimensions: learning science, teaching science, the science curriculum, science teachers and students of science teaching. The results show that teaching and learning science is the main problem addressed by most studies conducted in Latin America and the Caribbean. 


\section{Introducción}

Conocer el estado actual y las líneas de investigación prioritarias que se realizan en América Latina y el Caribe, en el área de las Didácticas de las Ciencias Naturales, permite conocer las problemáticas que preocupan y ocupan a la comunidad científica y educativa en la que estamos inmersos. Se convierte así en un conocimiento con el que resulta indispensable contar al momento de planificar, evaluar y reorientar las investigaciones en pos de que los resultados contribuyan a la generación de conocimientos que den respuestas a las preguntas planteadas por la comunidad científica. Este estudio pretende identificar, describir y caracterizar las principales líneas de investigación.

Este tipo de revisiones resulta especialmente importante en nuestra área dado el crecimiento exponencial de las publicaciones realizadas en los últimos años (Benarroch, 2010), y es necesario realizarlas ya que, salvo algunas excepciones (Consejo Mexicano de Investigaciones Educativas, 2003; Valbuena, Hernández, Correa, Amórtegui y Ruiz, 2008, 2010), la mayoría de las revisiones hasta el momento hacen alusión principalmente a contextos diferentes. Como afirman Pro y Rodríguez (2011), dichos balances no son trasladables a nuestras realidades, por lo que consideramos indispensable sumar a los análisis realizados uno que contemple nuestro entorno.

La Didáctica de las Ciencias irrumpe como disciplina a finales de la década de los ochenta. Porlán (1998), a fines de los noventa, la reconoce como una "disciplina práctica emergente que se incluye en el campo más amplio de las Ciencias de la educación [...] cuyo objeto de estudio son los sistemas de enseñanzaaprendizaje". Este autor propone desde entonces que la finalidad de la Didáctica de las Ciencias es "describir y analizar los problemas más significativos de la enseñanza-aprendizaje de las ciencias y elaborar y experimentar modelos que, a la luz de los problemas detectados, ofrezcan alternativas prácticas fundamentadas y coherentes" (p. 28).

Estudios epistemológicos, históricos y sociológicos realizados por Adúriz e Izquierdo (2002) sostienen la autonomía de la Didáctica de las Ciencias y describen las etapas por las que ha pasado hasta constituirse en una disciplina autónoma incluida dentro de las Ciencias Sociales.

Los avances en la investigación en educación en Ciencias, sumado a la consolidación de grupos y proyectos de investigación y la posibilidad de difundir y conocer rápida y masivamente los resultados de investigación de estos grupos, conllevó a que la didáctica de las ciencias se consolidara como una disciplina científica, metodológica y teóricamente fundamentada.

En ese andar, Moreira (2004) vislumbra que la investigación en educación en Ciencias evolucionó de la siguiente manera:

- Grandes proyectos curriculares en los años 50 y 60

- Estudios sobre concepciones alternativas en los años 70

- Estudios sobre cambio conceptual en los años 80

- Estudios sobre representaciones mentales en los años 90

- Estudios relacionados con el profesor de Ciencias y estudios microetnográficos en los últimos años

Y reconoce que las líneas de investigación que han producido (y siguen produciendo) conocimientos sobre educación en Ciencias están relacionadas con: métodos/técnicas/estrategias de enseñanza; conocimiento intuitivo de los estudiantes; aprendizaje de las Ciencias (principalmente en relación al cambio conceptual); currículum de Ciencias; formación/conocimiento/accionar de los profesores de Ciencias; contexto en que se da la educación en Ciencias; las nuevas tecnologías en la enseñanza de las Ciencias; la evaluación (del aprendizaje, del currículum, de la enseñanza).

En concordancia con ello, Gallego (2004) propone como campos de saber y de investigación delimitados por la didáctica de las Ciencias Naturales: la formación inicial y continua de profesores de Ciencias; las concepciones epistemológicas (e históricas), pedagógicas y didácticas de los profesores de Ciencias; las estrategias de enseñanza y las ideas alternativas del estudiantado; la confiabilidad de los textos de 
enseñanza; el problema de la evaluación; las relaciones CTSA (Ciencias, Tecnología, Sociedad y Ambiente); las intencionalidades curriculares.

Podemos hoy considerar a la Didáctica de las Ciencias como la disciplina que aborda y resuelve problemas relacionados con la enseñanza y el aprendizaje de las Ciencias. Y las líneas de investigación prioritarias que consolidan y acrecientan su cuerpo de conocimiento estarían relacionadas con:

- Aprendizaje de las Ciencias: conocimiento de los estudiantes-procesos de aprendizaje de las Ciencias-evaluación del aprendizaje.

- Enseñanza de las Ciencias: recursos/estrategias/métodos/técnicas-evaluación de la enseñanza.

- Currículum en Ciencias: innovaciones-evaluación.

- Profesorado en Ciencias: conocimiento; formación inicial y continua-accionar didáctico del docente en ejercicio.

Este trabajo busca analizar cuáles de estas líneas se están desarrollando en mayor medida en América Latina y el Caribe, y analizar si existen otras emergentes.

\section{Metodología}

En este estudio se analizan revistas científicas publicadas en Latinoamérica y el Caribe con acceso libre, en línea. En los últimos años, y gracias a las Tecnologías de la Información y Comunicación (Tic), ha aumentado notablemente el número de revistas académicas publicadas en línea (e indexadas en las principales bases de datos que reúnen a las revistas científicas), que posibilitan el libre acceso a los artículos publicados. Y también es creciente el uso de estas revistas en la comunidad académica, justamente por la posibilidad de acceder en forma libre y gratuita.

Analizar este tipo de revistas permite acceder a un vasto número de artículos y con ello conformar una base documental que sea representativa; para garantizar que la base documental incluya a todos los países que editan este tipo de revistas, se definen los siguientes criterios para su selección:

1) Las revistas seleccionadas deben estar indexadas en las principales bases de datos que integran publicaciones de países latinoamericanos y del Caribe sobre Educación e Investigación Educativa (Latindex, OEI-CREDI, Dialnet, CSIC, Redalyc, Scielo) en el área Ciencias Sociales y sub-área Educación.

2) En su título deben aparecer palabras clave relacionadas con la Investigación en Educación científica (por ejemplo: Ciencias, Ciencias Exactas, Ciencias Naturales, Física, Química, Biología, Investigación, Investigación Educativa, Didáctica de las Ciencias, escuela, etc.).

3) Todos los países de Latinoamérica y el Caribe que editan este tipo de revistas deben estar representados con al menos una publicación. En el caso de países que contaran con más de una revista relacionada con la Investigación Educativa (como es el caso de Argentina y Brasil) se eligen todas las especializadas en la didáctica de las Ciencias Experimentales (o disciplinas que las constituyen).

A fin de asegurar que el análisis revele una tendencia en las líneas sobre las que se están investigando, se analizan los trabajos publicados (disponibles en Internet) durante diez años (correspondiente al período 2004-2013). De ellos se eligen sólo aquellos que reporten investigaciones educativas y proporcionen datos empíricos sobre aspectos relacionados con el aprendizaje y la enseñanza de las Ciencias en los distintos niveles educativos (excluyéndose los que realizan aportes teóricos o metodológicos o que proponen revisiones bibliográficas y los que presentan innovaciones didácticas o experiencias áulicas que sólo describen metodologías de enseñanza pero no aportan datos de investigación inherente, por ejemplo, al impacto de la innovación sobre el aprendizaje propiciado, a las características del aprendizaje experimentado por los alumnos, al rol-accionar-metodología-perfil didáctico del docente, etc.). La base documental quedó conformada por 15 revistas (ver tabla I) y 490 artículos. 
Tabla I. Base documental

\begin{tabular}{l|l}
\hline País de edición & Título de la revista \\
\hline Argentina & $\begin{array}{l}\text { Revista Electrónica de Investigación en Educación en Ciencias } \\
\text { Revista de Enseñanza de la Física } \\
\text { Revista de Educación en Biología } \\
\text { Revista de Adeqra }\end{array}$ \\
\hline Brasil & $\begin{array}{l}\text { Investigações em Ensino de Ciência } \\
\text { Revista Brasileira de Pesquisa em Educação em Ciências } \\
\text { Caderno Brasileiro de Ensino de Física }\end{array}$ \\
\hline Chile & Estudios Pedagógicos \\
\hline Costa Rica & Revista Electrónica Actualidades Investigativas en Educación \\
\hline Cuba & Revista Didasc@lia: Didáctica y Educación \\
\hline México & $\begin{array}{l}\text { Latin American Journal of Physics Education } \\
\text { Revista Electrónica de Investigación Educativa } \\
\text { Revista Mexicana de Investigación Educativa }\end{array}$ \\
\hline Puerto Rico & Cuaderno de Investigación en la Educación \\
\hline Venezuela & Revista Universitaria Arbitrada de Investigación y Diálogo Académico \\
\hline
\end{tabular}

Con el fin de recolectar datos que permitan limitar las líneas de investigación prioritarias, se analizan el título y resumen de los artículos seleccionados y se identifican aquellas palabras claves y preposiciones que evidencien el problema de investigación abordado. Para organizar y sistematizar los datos recolectados se establecieron dimensiones, subdimensiones y categorías de análisis, atendiendo a las líneas de investigación que, según lo analizado con antelación, contribuyen a la consolidación y crecimiento del cuerpo de conocimiento de la didáctica de las Ciencias Naturales. Así se definen las unidades de análisis: Aprendizaje de las Ciencias, Enseñanza de las Ciencias, Currículum en Ciencias, Profesorado en Ciencias, Estudiantes de Profesorado en Ciencias y Otros temas.

En la dimensión Aprendizaje de las Ciencias se incluyen trabajos que analizan el conocimiento de los alumnos, diferenciando los que indagan su saber espontáneo y con ello concepciones, modos de razonar, argumentar, explicar, resolver situaciones problemáticas y realizar tareas experimentales (subdimensión Conocimiento de los alumnos) de los que estudian o miden alguna variable asociada con el aprendizaje conceptual, procedimental y actitudinal experimentado (subdimensión Aprendizaje de los estudiantes).

En Enseñanza de las Ciencias se incluyen artículos que evalúan el impacto de recursos o metodologías didácticas que consideradas innovadoras e intentan superar una determinada problemática, o por ser un recurso didáctico que no ha sido implementado previamente como tal, o que su impacto no ha sido medido mediante un trabajo de investigación. Así, la subdimensión Estrategias o recursos didácticos contiene artículos que evalúan el impacto sobre la enseñanza o el aprendizaje de recursos didácticos o estrategias didácticas, mientras que la Propuesta de enseñanza abarca trabajos que analizan el impacto de una metodología de enseñanza diseñada e implementada en la investigación.

En la dimensión Currículum en Ciencias se incluyen artículos que analizan diseños curriculares o evalúan el impacto de innovaciones curriculares.

Dentro de la dimensión Profesorado en Ciencias se definen las subunidades Conocimiento y accionar del profesor en ejercicio y Formación docente continua. La primera engloba trabajos que estudian las concepciones del profesor sobre alguna temática científica; su conocimiento pedagógico de ciertos contenidos; sus opiniones sobre alguna variable que caracterizan los procesos de enseñanza y aprendizaje; su accionar (y con ello las estrategias que pone en juego en el aula; el discurso que implementa al interaccionar con los alumnos; los modelos didácticos subyacentes), mientras que la segunda subunidad reúne trabajos que dan cuenta del impacto de distintas capacitaciones docentes implementadas sobre el aprendizaje científico-didáctico propiciado en los docentes.

Estas mismas subunidades se repiten en la dimensión Estudiantes de profesorado en Ciencias, siendo los alumnos de las carreras de profesorado el objeto de estudio en estos casos. 
Por último, la dimensión Otros temas incluye artículos variados que no corresponden a las unidades anteriores y que por la frecuencia con que se encuentran no son suficientes para crear una nueva dimensión.

En la tabla II se resumen las dimensiones (A, B... X); subdimensiones (X1, X2 ... Xn) y categorías seleccionadas $(1,2 \ldots n)$ y el tipo de trabajos que se agrupan en cada una de ellas.

Tabla II. Dimensiones, subdimensiones y categorías definidas

\begin{tabular}{|c|c|c|}
\hline \multicolumn{3}{|c|}{ A. Aprendizaje de las Ciencias } \\
\hline \multicolumn{2}{|r|}{ A.1 Características del saber de los estudiantes } & \multirow{4}{*}{$\begin{array}{l}\text { Trabajos que indagan concepciones; modos de } \\
\text { razonar, argumentar, explicar, resolver situaciones } \\
\text { problemáticas y/o realizar tareas experimentales }\end{array}$} \\
\hline 1 & Conocimiento conceptual & \\
\hline 2 & $\begin{array}{l}\text { Conocimiento procedimental (habilidades y destrezas } \\
\text { manuales y cognitivas) }\end{array}$ & \\
\hline 3 & Actitudes-Emociones & \\
\hline \multicolumn{2}{|r|}{ A.2 Aprendizaje de los estudiantes } & \multirow{5}{*}{$\begin{array}{l}\text { Trabajos que indagan el aprendizaje conceptual, } \\
\text { procedimental y/o actitudinal y/o miden alguna } \\
\text { variable asociada con estos procesos. }\end{array}$} \\
\hline 1 & Construcción del conocimiento conceptual & \\
\hline 2 & Construcción del conocimiento procedimental & \\
\hline 3 & Construcción del conocimiento actitudinal & \\
\hline 4 & Estrategias y estilos de aprendizaje-Metacognición & \\
\hline \multicolumn{3}{|c|}{ B. Enseñanza de las Ciencias } \\
\hline \multicolumn{2}{|r|}{ B.1 Estrategias o Recursos didácticos } & \multirow{3}{*}{$\begin{array}{l}\text { Artículos que evalúan el impacto sobre la enseñanza } \\
\text { o el aprendizaje de estrategias o recursos didácticos. }\end{array}$} \\
\hline 1 & Implementación de estrategias didácticas & \\
\hline 2 & Implementación de recursos didácticos & \\
\hline \multicolumn{2}{|r|}{ B.2 Propuestas de enseñanza } & \multirow{4}{*}{$\begin{array}{l}\text { Trabajos que analizan el impacto de propuestas de } \\
\text { enseñanza. }\end{array}$} \\
\hline 1 & Implementación y evaluación propuestas didácticas & \\
\hline 2 & $\begin{array}{l}\text { Implementación y evaluación propuestas de educación } \\
\text { a distancia }\end{array}$ & \\
\hline 3 & $\begin{array}{l}\text { Implementación y evaluación propuestas didácticas áulicas } \\
\text { tradicionales }\end{array}$ & \\
\hline \multicolumn{3}{|c|}{ C. Currículum en Ciencias } \\
\hline 1 & $\begin{array}{l}\text { Implementación y evaluación de proyectos curriculares, } \\
\text { cambio de planes de estudio }\end{array}$ & \multirow{3}{*}{$\begin{array}{l}\text { Artículos que analizan diseños curriculares y/o } \\
\text { evalúan el impacto de innovaciones curriculares. }\end{array}$} \\
\hline 2 & $\begin{array}{l}\text { Inserción de tópicos de ciencia contemporánea en el } \\
\text { currículum }\end{array}$ & \\
\hline 3 & Análisis en función de distintas variables & \\
\hline \multicolumn{3}{|c|}{ D. Profesorado en Ciencias } \\
\hline \multicolumn{2}{|r|}{ D. 1 Conocimiento y accionar del profesor en ejercicio } & \multirow{10}{*}{$\begin{array}{l}\text { Artículos relacionados con la formación continua del } \\
\text { profesorado en Ciencias }\end{array}$} \\
\hline 1 & Conocimiento del profesor & \\
\hline 2 & Saber procedimental científico & \\
\hline 3 & Conocimiento pedagógico del contenido & \\
\hline 4 & Accionar docente didáctico & \\
\hline 5 & Accionar docente comunicativo & \\
\hline 6 & Modelos didácticos subyacentes a las prácticas & \\
\hline \multicolumn{2}{|r|}{ D.2 Formación docente continua } & \\
\hline 1 & Implementación y/o evaluación propuestas de formación & \\
\hline 2 & $\begin{array}{l}\text { Aprendizaje ante la implementación de una propuesta de } \\
\text { formación }\end{array}$ & \\
\hline \multicolumn{3}{|c|}{ E. Estudiantes de profesorado en Ciencias } \\
\hline \multicolumn{2}{|r|}{ E.1 Conocimiento y accionar del profesor en formación } & \multirow{10}{*}{$\begin{array}{l}\text { Artículos relacionados con la formación docente } \\
\text { inicial }\end{array}$} \\
\hline 1 & Conocimiento conceptual & \\
\hline 2 & Conocimiento procedimental & \\
\hline 3 & Conocimiento actitudinal & \\
\hline 4 & Accionar docente didáctico & \\
\hline 5 & Accionar docente comunicativo & \\
\hline 6 & Modelos didácticos & \\
\hline \multicolumn{2}{|r|}{ E.2 Formación docente inicial } & \\
\hline 1 & Implementación y/o evaluación propuestas de formación & \\
\hline 2 & $\begin{array}{l}\text { Aprendizaje ante la implementación de una propuesta de } \\
\text { enseñanza }\end{array}$ & \\
\hline \multicolumn{3}{|c|}{ F. Otros temas } \\
\hline
\end{tabular}


Una vez categorizados los artículos analizados se calcula la frecuencia de representatividad de cada dimensión, subdimensión y categoría a fin de dilucidar la tendencia en las líneas de investigación que se están llevando adelante. Cuando los trabajos analizados no pueden asociarse a las dimensiones previamente definidas se les incluye en una dimensión "Otros temas". Es importante aclarar que hay artículos que fueron incluidos en más de una categoría, por lo que la suma de las frecuencias informadas puede exceder el total de artículos analizados.

\section{Resultados}

En la tabla III se muestra la frecuencia de artículos agrupados en las distintas dimensiones de análisis, resalta en sombreado aquella que se halla representada con mayor número de trabajos.

Tabla III. Número de artículos distribuidos en las distintas dimensiones

\begin{tabular}{lc}
\hline Dimensiones & Artículos \\
\hline A. Aprendizaje de las Ciencias & 133 \\
B. Enseñanza de las Ciencias & 240 \\
C. Currículum en Ciencias & 17 \\
D. Profesorado en Ciencias & 91 \\
E. Estudiantes de profesorado en Ciencias & 49 \\
\hline
\end{tabular}

Este primer análisis permite apreciar que las líneas de investigación prioritarias se refieren a la Enseñanza de las Ciencias. Al hacer un análisis intra-dimensión se observa, tal como lo muestra la tabla IV, que la mayoría de los artículos se refieren a la implementación y evaluación de propuestas didácticas innovadoras. 
Tabla IV. Número de artículos agrupados en subdimensiones y categorías de análisis definidas en relación a la Enseñanza y al Aprendizaje de las Ciencias

\begin{tabular}{|c|c|c|}
\hline \multicolumn{2}{|c|}{ Subdimensiones } & Artículos \\
\hline \multicolumn{2}{|c|}{ A.1Características de los estudiantes } & 66 \\
\hline 1 & Conocimiento conceptual & 47 \\
\hline 2 & Conocimiento procedimental & 11 \\
\hline 5 & Conocimiento actitudinal & 8 \\
\hline \multicolumn{2}{|r|}{ A.2 Aprendizaje de los estudiantes } & 67 \\
\hline 1 & Construcción del conocimiento conceptual & 40 \\
\hline 2 & Construcción del conocimiento procedimental & 8 \\
\hline 3 & Construcción del conocimiento actitudinal & 2 \\
\hline 4 & Estrategias de aprendizaje-Metacognición & 17 \\
\hline \multicolumn{2}{|r|}{ B.1 Estrategias o Recursos didácticos } & 166 \\
\hline 1 & Tecnológicos & 13 \\
\hline 2 & Libros de textos & 62 \\
\hline 3 & Sitios y/o actividades de educación no formal & 4 \\
\hline 4 & Lectura & 2 \\
\hline 5 & Resolución de problemas & 13 \\
\hline 6 & Pre-exámenes & 2 \\
\hline 7 & Diseño de experiencias & 6 \\
\hline 8 & Metodología de la investigación & 5 \\
\hline 9 & Tareas multipasos & 1 \\
\hline 10 & Analogías/metáforas & 6 \\
\hline 11 & Juegos & 3 \\
\hline 12 & Elaboración de textos & 1 \\
\hline 13 & Herramientas "cognitivas" & 3 \\
\hline 14 & Modelos & 8 \\
\hline 15 & Interacciones sociales: discurso & 17 \\
\hline 16 & Trabajo cooperativo & 1 \\
\hline 17 & CTS & 4 \\
\hline 18 & Videos & 3 \\
\hline 19 & Historietas & 5 \\
\hline 20 & Imaginación & 1 \\
\hline 21 & Representaciones/maquetas/Imágenes & 6 \\
\hline \multicolumn{2}{|r|}{ B.2 Propuestas de enseñanza } & 74 \\
\hline 1 & $\begin{array}{l}\text { Implementación y evaluación propuestas } \\
\text { didácticas áulicas innovadoras }\end{array}$ & 64 \\
\hline 2 & $\begin{array}{l}\text { Implementación y evaluación propuestas de } \\
\text { educación a distancia }\end{array}$ & 8 \\
\hline 4 & $\begin{array}{l}\text { Implementación y evaluación propuestas } \\
\text { didácticas áulicas tradicionales }\end{array}$ & 2 \\
\hline
\end{tabular}

Se observa también que hay una marcada tendencia hacia el análisis y evaluación del impacto de recursos o estrategias didácticas. Puntualmente, se halla un alto número de artículos que tiene a los libros de textos como objeto de estudio y, en la mayoría de ellos, se realiza un análisis científico o didáctico de su contenido.

En cuanto a la evaluación del impacto de resultados didácticos, los más evaluados, aunque con frecuencias bajas, son los tecnológicos. En tanto las estrategias didácticas más evaluadas resultan ser las discursivas y las que involucran la resolución de problemas como estrategia central de enseñanza.

La segunda línea de investigación en orden de representatividad se refiere al Aprendizaje de las Ciencias. Al respecto se percibe que el eje central es el conocimiento espontáneo de los alumnos y los procesos de aprendizaje que suceden en la educación formal. Estos estudios estarían centrando la atención en el saber que comparten los alumnos previo a la enseñanza formal (caracterizado como concepciones alternativas, representaciones cognitivas, representaciones sociales, modelos mentales, modos de conocer, teorías implícitas, según el marco teórico de referencia) y en el proceso de construcción de dicho conocimiento conceptual (analizado en término de cambio conceptual, desarrollo conceptual, desarrollo cognitivo, aprendizaje significativo, cambio de idea, cambio de rendimiento, según el marco teórico subyacente). 
En segundo término, y con frecuencias mucho menores, se encuentran los trabajos que abordan el saber procedimental de los alumnos (relacionado principalmente con la forma en que elaboran argumentaciones) y aspectos intrínsecos al proceso de aprendizaje (estrategias de aprendizaje implementadas y habilidades de metacognición desarrolladas). El resto de las subdimensiones están representadas con un número muy reducido de trabajos.

Se advierte, como lo muestra la tabla $V$, que la mayoría de estos trabajos se refieren a la enseñanza de la Física en el nivel secundario, aunque hay un número no despreciable de artículos que se ocupan de la educación universitaria, lo que implicaría que se empiezan a atender a sus problemáticas educativas.

Tabla V. Número de artículos de enseñanza-aprendizaje agrupados según nivel educativo, disciplina o área científica

\begin{tabular}{l|c|l|c}
\hline Nivel educativo & Artículos & \multicolumn{1}{|c|}{ Disciplina o área } & Artículos \\
\hline Preescolar & 2 & Química & 75 \\
Primario & 20 & Física & 183 \\
Secundario & 169 & Astronomía & 5 \\
Universitario & 133 & Biología/Ecología/Medio & 55 \\
& & Ambiente & 33 \\
\hline
\end{tabular}

Otra de las líneas de investigación prioritarias tiene como objeto de estudio al profesorado de Ciencias en ejercicio o en formación, tal como lo refleja la tabla VI.

Tabla VI. Número de artículos agrupados en las distintas categorías definidas en relación al profesorado en Ciencias y a los estudiantes de profesorado en Ciencias

\begin{tabular}{|c|c|c|}
\hline \multicolumn{2}{|c|}{ Profesorado en Ciencias } & Artículos \\
\hline \multicolumn{2}{|r|}{ D.1 Pensamiento y accionar del profesor en ejercicio } & 80 \\
\hline 1 & Conocimiento y pensamiento & 40 \\
\hline 2 & Saber procedimental científico & 2 \\
\hline 3 & Conocimiento pedagógico del contenido & 4 \\
\hline 4 & Accionar docente didáctico & 14 \\
\hline 5 & Accionar docente comunicativo & 9 \\
\hline 6 & Modelos didácticos & 4 \\
\hline \multicolumn{2}{|c|}{ D.2 Formación docente continua } & 11 \\
\hline 1 & Implementación y/o evaluación propuestas de formación & 7 \\
\hline 2 & Aprendizaje ante la implementación de una propuesta de formación & 4 \\
\hline \multicolumn{3}{|c|}{ Estudiantes de Profesorado en Ciencias } \\
\hline \multicolumn{2}{|r|}{ E.1 Pensamiento y accionar del profesor en formación } & 25 \\
\hline 1 & Conocimiento conceptual & 15 \\
\hline 2 & Conocimiento procedimental & 0 \\
\hline 3 & Conocimiento actitudinal & 0 \\
\hline 4 & Accionar docente didáctico & 6 \\
\hline 5 & Accionar docente comunicativo & 4 \\
\hline 6 & Modelos didácticos & 0 \\
\hline \multicolumn{2}{|c|}{ E.2 Formación docente inicial } & 27 \\
\hline 1 & Implementación y/o evaluación propuestas de formación & 13 \\
\hline 2 & Aprendizaje ante la implementación de una propuesta de formación & 14 \\
\hline
\end{tabular}

Como puede observarse, la problemática más atendida en relación a los docentes de Ciencias implica el análisis de su conocimiento y pensamiento. Así, la mayoría de los artículos agrupados en esta dimensión estudia las concepciones de los profesores desde múltiples y diversas temáticas: idea de ciencia, concepciones sobre alguna temática científica, sobre la enseñanza, sobre el aprendizaje, sobre algún recurso didáctico, sobre su propia práctica, entre otras.

El resto de las unidades de análisis están representadas con un número bajo (o nulo) de artículos, resultando entre ellas la más significativa la que implica el estudio del accionar docente cuando desarrolla su tarea en el aula y al enseñar algún tópico científico. 
En cuanto a la formación docente, es reducido el número de artículos que se ocupan de evaluar las propuestas de formación continua y no se estaría analizando la manera en que dichas propuestas impactan sobre el saber de los profesores.

En relación a la formación inicial de los futuros profesores se advierte, en cambio, que el número de artículos que estudian el saber de estos estudiantes es equivalente al número de trabajos que analizan el impacto de propuestas de enseñanza innovadoras.

Finalmente, la dimensión Currículum en Ciencias está representada por un reducido número de trabajos, la mayoría de ellos enfocados en cómo influyen distintas variables sobre el currículum (como la relación entre cambios de los planes de estudio de profesorados y la calidad y cantidad de egresados, la incorporación de la dimensión ambiental en los currículo de profesorados, entre otros).

Tabla VII. Número de artículos agrupados en las distintas categorías definidas en relación al Currículum en Ciencias

\begin{tabular}{l|l|c}
\hline Currículum en Ciencias & Artículos \\
\hline 1 & Implementación y evaluación de proyectos curriculares, análisis de planes de estudio, & 7 \\
2 & cambio de planes de estudio & 3 \\
3 & Inserción de tópicos de ciencia contemporánea en el currículum & 7 \\
\hline
\end{tabular}

Del total de artículos analizados, 21 se incluyen en la dimensión Otros temas, ya que se refieren a situaciones muy concretas (no asociables a las categorías definidas), como opiniones de distintos actores del proceso educativo (alumnos, padres, investigadores) sobre diversas temáticas, impacto de las experiencias científicas extraescolares sobre el aprendizaje formal de las Ciencias; inclusión de estudiantes con dificultades auditivas o visuales, entre las más representativas. Cabe resaltar que estas temáticas están representadas por un número reducido de trabajos.

\subsection{Análisis de resultados}

Los resultados obtenidos permiten concluir que el problema central de las investigaciones publicadas en revistas de América latina y el Caribe radica en cómo enseñar Ciencias. En tal sentido, la mayoría de los trabajos analizados estudian el impacto de propuestas de enseñanza en entornos educativos tradicionales. Son muy pocos los que estudian propuestas de enseñanza a distancia, a pesar de que existe una tendencia mundial a utilizar los nuevos medios de comunicación para desarrollar cursos y carreras de grado y posgrado de forma no presencial. Cómo implementar estas propuestas y cómo impactan sobre el aprendizaje aún está lejos de convertirse en una línea de investigación prioritaria.

En el mismo sentido, si bien son varios los recursos didácticos que están evaluándose, los tecnológicos (como las TIC, simulaciones, software) recién comienzan a ser estudiados, pese a su rápida y creciente inclusión en las vidas de los alumnos en general y en las aulas de ciencia en particular. Moreira (2004) y Cachapuz, Lopes, Paixão, Praia y Guerra (2006) expresan que entre las líneas prioritarias a futuro, están justamente las TIC en la educación.

La otra línea prioritaria encontrada en esta investigación estudia las concepciones de los alumnos (principalmente de nivel secundario) y el cambio-construcción conceptual que se produce cuando se implementan en el aula propuestas de enseñanza innovadoras.

Si bien se encuentran algunos artículos que se refieren al "saber hacer" de los estudiantes, principalmente relacionado con la elaboración de argumentaciones, no sería todavía ésta una línea prioritaria, aunque lo es ya en investigaciones realizadas en Europa (Benarroch, 2010).

Respecto al nivel educativo y la disciplina en torno a la cual se estudian los procesos de enseñanza y aprendizaje, la Física y la Educación Secundaria son el foco de las investigaciones que se realizan actualmente en América Latina y el Caribe, tendencia histórica en el ámbito de la investigación en Didáctica de las Ciencias Naturales. No obstante, hay indicios de una creciente preocupación sobre las 
problemáticas que suceden en el nivel universitario, y se observa también una paulatina inclusión de las problemáticas ambientales como temática científica de interés (tendencia mundial en la investigación y educación científica).

Por último, la línea de investigación que atañe a la formación del profesorado en general y a la formación inicial en particular, no sería aún una línea prioritaria. En correspondencia con ello, Moreira (2004) sostiene que hay poca transferencia al aula del conocimiento producido por la investigación educativa en Ciencias y que sería conveniente la participación de profesores en grupos de investigación y la inclusión de la investigación en Ciencias en el currículum de la formación inicial del profesorado.

Dado los resultados obtenidos, y las temáticas que parecerían ser prioritarias, se considera que si bien es necesario profundizar al respecto, resultaría oportuno comenzar a indagar en las líneas de vanguardia a nivel mundial (como el uso de TIC como medios de representación externo; las relaciones CTSA; el conocimiento científico-didáctico-tecnológico-pedagógico del docente de Ciencias) para contextualizarlas en nuestras realidades educativas y realizar aportes concretos al cuerpo de conocimiento que se está construyendo en la comunidad científica, así como crear ámbitos que propicien la participación en investigación de los profesores en ejercicio y que contribuyan a fomentar la reflexión respecto a la importancia de incorporar en las prácticas áulicas los resultados de investigación.

Respecto de estos ámbitos, en la Facultad de Ingeniería de la Universidad Nacional del Centro de la Provincia de Buenos Aires, existe ya hace varios años el Grupo Operativo en Didáctica de las Ciencias Experimentales (GODCE), que reúne a docentes investigadores de esa institución y a docentes de los niveles educativos primario y secundario. El grupo se ha constituido en un innegable aporte a la formación continua y permanente de los docentes en servicio y resulta, además, una herramienta fundamental para el acompañamiento de docentes noveles (Rocha, Bertelle, Iturralde, García de Cajén, Roa, Fuhr Stoessel y Boucíguez, 2012). Las principales actividades que se realizan en este contexto son: 1) Talleres de discusión sobre contenidos de Enseñanza de las Ciencias, en los que se comparten los principales resultados de la investigación educativa en relación con la/s temática/s central/es que se aborda/n en cada taller; 2) Elaboración de propuestas innovadoras contextualizadas en la realidad educativa de los participantes, y 3) Acompañamiento por parte de los investigadores al docente y los estudiantes en el aula, mientras trabajan con las propuestas elaboradas, para observar, registrar datos y apoyar el desarrollo, en una estrategia de trabajo cooperativo-encuentros de reflexión y de discusión: en los que se analizan los resultados de los trabajos en aula, con todo el grupo de docentes e investigadores participantes. Se genera así un ámbito de interacción y de construcción de conocimiento que alimenta directamente a la formación de profesores.

\section{Conclusiones}

Más allá de los resultados encontrados, este trabajo permitió evidenciar que hay una gran cantidad de investigaciones llevándose a cabo en Latinoamérica y el Caribe, lo que denota que se está trabajando y creciendo en el campo de la Didáctica de las Ciencias Experimentales en la región. Se espera que los resultados aportados permitan seguir avanzando, redefiniendo, profundizando y proyectando nuevas líneas de investigación que permitan, cada vez con mayor fundamento y rigor metodológico, entender cómo aprenden ciencias los estudiantes de distintos niveles educativos y cuáles son los currículos, recursos, formación, accionar y estrategias docente, que más y mejor favorecen dicho aprendizaje.

\section{Referencias}

Adúriz-Bravo, A. e Izquierdo, M. (2002). Acerca de la didáctica de las ciencias como disciplina autónoma. Revista Electrónica de Enseñanza de las Ciencias, 1(3), 130-140.

Benarroch, A. (2010). La investigación en Didáctica de las Ciencias Experimentales en las etapas educativas de infantil y primaria. Documento presentado en el XXIV Encuentro de Didáctica de las Ciencias Experimentales. Jaén, España. 
Cachapuz, A. F., Lopes, B., Paixão, F., Praia, J. F. y Guerra, C. (2006). Reseña de Seminario Internacional sobre "El estado actual de la Investigación en Enseñanza de las Ciencias". Eureka, 3(1), 167-171.

Consejo Mexicano de Investigaciones Educativas. (2003) La investigación educativa en México: usos y coordinación. Revista Mexicana de Investigación Educativa, 8(19), 847-898.

Gallego, R. (2004). Un concepto epistemológico de modelo para la didáctica de las ciencias experimentales. Revista Electrónica de Enseñanza de las Ciencias, 3(3), 301-319.

Moreira, M. (2004). Investigación básica en educación en ciencias: una visión personal. Revista Chilena de Educación Científica, 3(1), 10-17.

Porlán, R. (1998). Pasado, presente y futuro de la didáctica de las ciencias. Enseñanza de las Ciencias, 16, 175-185.

Pro Bueno, A. y Rodríguez, J. (2011). La Investigación en la didáctica de las ciencias experimentales. Educatio Siglo XXI, 29(1), 129-148.

Rocha, A., Bertelle, A., Iturralde, C., García de Cajén, S., Roa, M., Fuhr Stoessel, A. y Boucíguez, M. (2013). Formación de Profesor de Química en la Universidad Nacional del Centro de la Provincia de Buenos Aires (Argentina). Eureka, 10(Núm. Extraordinario), 836-845.

Valbuena, E., Hernández, A., Correa, M., Amórtegui, E. y Ruiz, D. (2008). Estado del arte del campo de conocimiento de la Enseñanza de la Biología. Fase I. Proyecto de investigación. Bogotá: cIup-Universidad Pedagógica Nacional.

Valbuena, E., Hernández, A., Correa, M., Amórtegui, E. y Ruiz, D. (2010). Estado del Arte del campo de conocimiento de la Enseñanza de la Biología. Fase II. Proyecto de investigación. Bogotá: cIup-Universidad Pedagógica Nacional. 